\title{
Robust evaluation of intermolecular FRET using a large Stokes shift fluorophore as a donor
}

Carmen Santana-Calvo§,1, Francisco Romero¹, Ignacio López-González¹ \& Takuya Nishigaki”,1 ${ }^{1}$ Departamento de Genética del Desarrollo y Fisiología Molecular, Instituto de Biotecnología, Universidad Nacional Autónoma de México (IBT, UNAM), Avenida Universidad 2001, Col. Chamilpa, C.P. 62210, Cuernavaca, Morelos, México ${ }^{\S}$ Current address: Instituto Gulbenkian de Ciência, Rua da Quinta Grande 6, 2780-156 Oeiras, Portugal

BioTechniques 65: 211-218 (October 2018) 10.2144/btn-2018-0041

Keywords: binding assay • fluorescent protein • intermolecular FRET • large Stokes shift

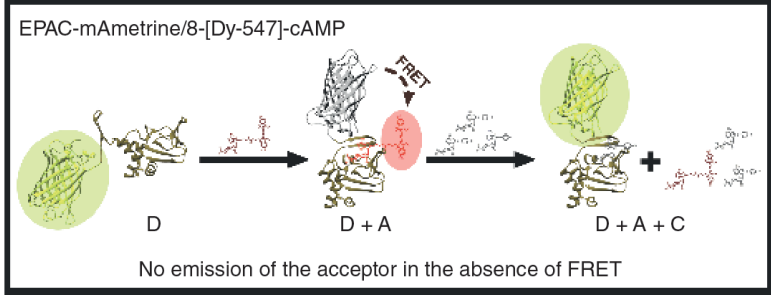

EPAC-CFP/8-Fluo-cAMP
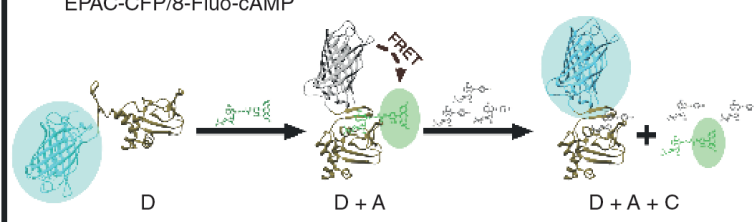

Emission of the acceptor even in the absence of FRET

Donor (D) Acceptor (A)

Fluorescence (or Förster) resonance energy transfer (FRET) is a straightforward and sensitive technique to evaluate molecular interactions. However, most of the popular FRET pairs suffer cross-excitation of the acceptor, which could lead to false positives. To overcome this problem, we selected a large Stokes shift (LSS) fluorophore as a FRET donor. As a successful example, we employed a new FRET pair mAmetrine (an LSS yellow fluorescence protein)/DY-547 (a cyanine derivative) to substitute CFP/fluorescein that we previously employed to study molecular interactions between cyclic nucleotidebinding domains and cyclic nucleotides. The new FRET pair is practically free of cross-excitation of the acceptor. Namely, a change in the fluorescence spectral shape implies evidence of FRET without other control experiments.

Fluorescence (or Förster) resonance energy transfer (FRET) has been used as an important tool to study molecular interactions since its efficiency is highly dependent on the distance between the two molecules as well as their relative orientation. Recently, we developed an intermolecular FRET-based binding assay for CAMP using cyan fluorescent protein (CFP) fused to the cyclic-nucleotide binding domain of EPAC1 (EPAC1 $\left.{ }_{\mathrm{CNBD}}-\mathrm{CFP}\right)$ as donor and fluorescein-labeled CAMP (8-(2-[Fluoresceinyl]aminoethylthio) adenosine-3',5'cyclic monophosphate, 8-Fluo-cAMP) (Supplementary Figure 1) as acceptor [1]. This FRET-based binding assay is more sensitive than other fluorescencebased methods such as fluorescence anisotropy [2] or fluorescence enhancement using the environment-sensitive fluorophore 8-(2-[7-Nitro-4-benzofurazanyl]aminoethylthio) adenosine-3',5'-cyclic monophosphate (8-NBD-cAMP) [3]. The high sensitivity of the FRET-based binding assay is due to the direct measurement of the fluorescence intensity of the bright donor (or the acceptor) being employed [1].

Despite its usefulness, this method suffers from cross-excitation of the acceptor, a well-known problem of almost every available FRET pair [4]. Namely, the excitation light of CFP (donor) signifi- cantly excites 8-Fluo-cAMP independently of FRET. In consequence, a part of 8-Fluo-cAMP emission is produced by direct excitation not only mediated through FRET [1]. Therefore, a simple fluorescence spectrum of the mixture of EPAC1 $1_{\mathrm{CNBD}}-\mathrm{CFP}$ and 8-Fluo-cAMP cannot tell us reliably if FRET occurs or not. To determine the degree of FRET between EPAC1 $1_{\mathrm{CNBD}}-\mathrm{CFP}$ and 8-Fluo-cAMP, we measured the fluorescence spectra in the absence and presence of excess amount of nonfluorescent cAMP analog (8-(4-Chlorophenylthio) adenosine3',5'-cyclic monophosphate [8-CPT-cAMP]) (Supplementary Figure 1), and determined the difference of the donor fluorescence

\section{METHOD SUMMARY}

We report a new fluorescence (or Förster) resonance energy transfer (FRET) pair that is free of cross-excitation of the acceptor by using a large Stokes shift fluorescent protein as donor. We used this advantage to study molecular interactions and developed a straightforward and reliable assay, based on intermolecular FRET, to monitor the binding of cyclic nucleotides to the protein domain CNBD in vitro. 
intensities between the two conditions [1]. It is also possible to confirm FRET by photobleaching of the acceptor with intensive illumination and subsequent fluorescence recovery of the donor [4], although it is difficult to determine precisely the degree of FRET with this method. When the quantum yield of the acceptor is high enough, there is a noninvasive method to evaluate the degree of FRET based on the cross-excitation of the acceptor, the so-called three-cube method $[5,6]$. However, this method requires a relatively complicated procedure to quantify a relative FRET efficiency and a certain calibration to determine an absolute FRET efficiency. Another straightforward and noninvasive method to determine the FRET efficiency is to measure the lifetime of the donor fluorophore [4]. However, this parameter cannot be obtained with a conventional spectrofluorometer.

In general, bright fluorophores have small Stokes-shift, namely a small difference between the excitation and emission peaks. Since an efficient FRET pair needs a large overlap between the emission spectrum of the donor and the excitation spectrum of the acceptor $(J[\lambda])$, cross-excitation of the acceptor upon the donor excitation is almost unavoidable. However, there are several large Stokes shift (LSS) fluorescent proteins (FP) with comparable brightness to EGFP: T-Sapphire, mAmetrine, LSSmOrange, mBeRFP and mCyRFP1 [7-10]. In theory, the use of a LSS-FP as a FRET donor should reduce cross-excitation of the acceptor without sacrificing the FRET efficiency. In actuality, this property was confirmed with T-Sapphire/DsRed pair as an intramolecular FRET pair [7,11].

In this study, we used mAmetrine [8,12], an LSS yellow FP, as a FRET donor and demonstrated an example of practically null cross-excitation of the acceptor upon the intermolecular FRET measurements. We propose that substitution of a FRET donor by an LSS fluorophore should reduce this cross-talk excitation, which would allow us to perform robust intermolecular FRET assays to study molecular interactions.

\section{Materials \& methods}

Materials

Plasmid encoding mAmetrine1.2 (pBADmAmetrine1.2) [12] was provided by Dr Robert Campbell (University of Alberta, Canada) through Addgene (plasmid
\#42171). We used mAmetrine1.2, since its photostability is about three-times improved compared with the original version of mAmetrine [8]. We call mAmetrine1.2 just mAmetrine in this manuscript for simplicity. The plasmid, pRSETB-EPAC1 ${ }_{\mathrm{CNBD}}$-CFP, was previously prepared in our group [1]. Cyclic AMP analogs 8-Fluo-cAMP, 8-(2-[DY-547] aminoethylthio) adenosine-3',5'-cyclic monophosphate (8-[DY-547]-AET-cAMP), and 8-CPT-cAMP were acquired from BioLog Life Science Institute (Bremen, Germany) through Axxora LLC. Their chemical structure is depicted in Supplementary Figure 1. Bacterial strains JM109(DE3) and BL21(DE3) were obtained from the National BioResource Project, and the Escherichia coli strain from the National Institute of Genetics (Mishima, Shizuoka). Restriction enzymes and ligase were purchased from Thermo Scientific $^{\top M}$ (Waltham, MA, USA); Vent ${ }^{\circledR}$ DNA Polymerase from New England Biolabs (Ipswich, MA, USA) and the Protease Inhibitor Cocktail Tablet, cOmplete ${ }^{\mathrm{TM}}$ from Roche (Basel, Switzerland).

Construction of EPAC1 ${ }_{\mathrm{CNBD}^{-}}$ mAmetrine \& EPAC1 ${ }_{\mathrm{R} 279 \mathrm{E}^{-m}}{ }^{\mathrm{m}}$ Ametrine A DNA fragment encoding mAmetrine was obtained by PCR using the following oligonucleotides, 5' CCC GGA TCC GAT GGT GAG CAA GGG CGA GGA G 3' and 5' GGA AGC TTA CTT GTA CAG CTC GTC CAT G $3^{\prime}$. The product of the PCR was inserted into pRSETB-EPAC1 ${ }_{\mathrm{CNBD}^{-}}-\mathrm{CFP}$ [1] by substitution of CFP by mAmetrine using BamHI and HindllI as cloning sites. As a negative control of our experiments, we prepared a mutant of EPAC1 ${ }_{\mathrm{CNBD}}-\mathrm{mAmetrine}$ that does not interact with CAMP (EPAC1 ${ }_{\text {R279E }}$ mAmetrine) by substituting the arginine at the position 279 of human EPAC1 for glutamic acid [1].

\section{Recombinant proteins}

expression \& purification

For bacterial expression, the E. coli BL21(DE3) strain was transformed with the plasmid of interest and one colony of the freshly transformed bacteria was used to inoculate $100 \mathrm{ml}$ of LB supplemented with ampicillin. Overexpression was achieved by growing the cultures at $20^{\circ} \mathrm{C}$ for $3-4$ days without induction by IPTG [13]. Cells were harvested by centrifugation $(2500 \times \mathrm{g}$, $20 \mathrm{~min}, 4^{\circ} \mathrm{C}$ ) and the cell pellet was frozen at $-80^{\circ} \mathrm{C}$ until further use.
To purify the recombinant proteins, the frozen pellet was re-suspended in $2 \mathrm{ml}$ of solution A (300 mM NaCl, 10 mM Tris- $\mathrm{HCl}$, $\mathrm{pH}$ 7.4). Protein was released by sonication in the presence of the protease inhibitor cOmplete cocktail; the lysate was then clarified by centrifuging at 20,000 $\times \mathrm{g}$ for $30 \mathrm{~min}$ at $4^{\circ} \mathrm{C}$. Since the $\mathrm{N}$ terminus of the expected proteins has a hexahistidine tag, the soluble protein fraction was loaded into a $1 \mathrm{~mL}$ Ni-NTA Superflow (Qiagen) chromatography column. Weakly bound proteins were washed by solution A with $50 \mathrm{mM}$ imidazole and the recombinant proteins were eluted by solution A with $250 \mathrm{mM}$ imidazole. Protein concentrations were determined by absorbance at maximum peak of each FPs: mAmetrine1.2 $\left(31,000 \mathrm{~cm}^{-1} \mathrm{M}^{-1}\right.$ at $\left.408 \mathrm{~nm}\right)$ [12] and CFP $\left(32,500 \mathrm{~cm}^{-1} \mathrm{M}^{-1}\right.$ at $\left.434 \mathrm{~nm}\right)$ [1]. Except where otherwise clearly indicated, the protein concentration used in the experiments was $50 \mathrm{nM}$; the aliquots were stored at $4^{\circ} \mathrm{C}$ prior to performing spectral characterization or binding assay.

\section{Fluorescence spectra acquisition}

Steady-state fluorescence excitation and emission spectra were recorded on an Olis upgraded Aminco SLM 8000 spectrofluorometer (On-Line Systems Inc., GA, USA). All the samples were prepared in recording buffer (150 mM NaCl, 10 mM Tris-HCl, pH 7.4) with a final volume of $2 \mathrm{ml}$ in disposable plastic cuvettes (Sigma-Aldrich) at room temperature. Spectra were collected with a $1 \mathrm{~nm}$ bandwidth and a dwell-time of $0.3 \mathrm{~s}$ per wavelength. The excitation and emission spectra of EPAC1 ${ }_{\mathrm{CNBD}^{-}}$ mAmetrine were obtained using a fixed emission wavelength at $525 \mathrm{~nm}$ and a fixed excitation wavelength at $408 \mathrm{~nm}$, respectively. Likewise, the fluorescence spectra of 8-[DY-547]-AET-cAMP were collected at $585 \mathrm{~nm}$ (for excitation) and $525 \mathrm{~nm}$ (for emission). The spectra showed represent the average of at least three measurements from three independent protein isolations or dye batch.

\section{FRET-based binding assay}

The emission spectrum of the samples comprising donor-only, acceptor-only, donor in presence of acceptor (D+Acceptor), and donor with acceptor in the presence of nonfluorescent competitor 8-CPT-cAMP ( $\mathrm{D}+\mathrm{A}+\mathrm{Comp}$ ) were collected as described in the previous paragraph but the excitation wavelength used was $408 \mathrm{~nm}$ for the 
experiments with EPAC1 $1_{C N B D}-m A$ metrine and 8-[DY-547]-AET-CAMP and $434 \mathrm{~nm}$ for EPAC1 ${ }_{\mathrm{CNBD}}$-CFP and 8-Fluo-CAMP. Different donor:acceptor ratios (1:2, 1:5, 1:10) were assessed using $50 \mathrm{nM}$ of EPAC $1_{\mathrm{CNBD}^{-}}$ mAmetrine and $100 \mathrm{nM}$ of EPAC $1_{\mathrm{CNBD}}$-CFP. The concentration of the competitor was 100-times higher than that of the acceptor. The FRET efficiency $(E)$ was calculated as $E=1-F_{\mathrm{DA}} / F_{\mathrm{D}}$; where $F_{\mathrm{DA}}$ is the fluorescence intensity measured at donor's peak wavelength of $D+$ Acceptor and $F_{\mathrm{D}}$, the fluorescence intensity at donor's peak wavelength in donor-only condition.

Determination of dissociation constant in equilibrium conditions

The dissociation constant $\left(\mathrm{K}_{\mathrm{d}}\right)$ between $\mathrm{EPAC1}_{\mathrm{CNBD}}$-mAmetrine and 8-[DY-547]-AETCAMP in equilibrium conditions was determined by measuring emission spectra of the mixture of EPAC1 $1_{\mathrm{CNBD}}-\mathrm{mAmetrine}$ and 8-[DY-547]-AET-CAMP in fixed molar ration (1:2) in different concentrations as previously reported [1]. In order to reduce the volume of the sample, we performed this experiment with $200 \mu$ l of the solution using 96-well plates (Greiner high and medium binding 96-well plates, black flat bottom, Sigma-Aldrich). As a highest concentration, $10 \mu \mathrm{M}$ of EPAC1 $1_{\mathrm{CNBD}}$-mAmetrine and $20 \mu \mathrm{M}$ 8-[DY-547]-AET-cAMP were prepared in solution $B$ (solution A with $0.1 \%$ BSA), then this solution was serially diluted by solution $B$ with dilution factor 3.16. The emission spectra of samples excited at $408 \mathrm{~nm}$ were determined with FS5 Spectrofluorometer with SC-40 96 Micro-well Plate Reader Cassette (Edinburgh Instruments, Livingston, UK). Spectra measurements of low concentration of samples were repeated according to an increase in the noise, and the average of spectra were used for further analysis. The net fluorescence intensities were obtained by subtraction of those of solution $\mathrm{B}$ alone. The ratio of fluorescence intensities at $525 \mathrm{~nm}$ (the peak of mAmetrine1.2) and $563 \mathrm{~nm}$ (isoemissive point) was plotted as function of the concentration of EPAC1 ${ }_{\mathrm{CNBD}}-\mathrm{mAmetrine}$. The $\mathrm{K}_{\mathrm{d}}$ value was determined by best fitting using the equation 1 as previously reported [1].

$$
-\frac{F_{525}}{F_{563}}=\alpha\left(\frac{3 x+K_{d}-\sqrt{\left(3 x+K_{d}\right)^{2}-8 x^{2}}}{2 x}\right)+\beta
$$

where $x$ is the total concentration of $\mathrm{EPAC}_{\mathrm{CNBD}}-\mathrm{mAmetrine}$ and $\alpha$ and $\beta$ are constants to allow the fitting. When $x$ is close to 0 (no interaction), Equation $1=\beta$. When $x$ is at infinity (the CNBD is 100\% occupied with the CAMP analogue), Equation $1=\alpha+\beta$. In our experiments, fluorescence spectra were quite noisy at a low concentration of $x$ (below $10 \mathrm{nM}$ ), therefore $\beta$ value was obtained using $100 \mathrm{nM}$ of EPAC1 ${ }_{\mathrm{CNBD}^{-}}$ mAmetrine in the presence of an excess of competitor (20 $\mu \mathrm{M}$ of 8-CPT-cAMP).

Determination of overlap integral

$(J[\lambda])$ \& Förster distance $\left(R_{0}\right)$

$J(\lambda)$ was obtained using the following equation [14] (Equation 2):

$$
J(\lambda)=\frac{\int F_{D}(\lambda) \varepsilon_{A}(\lambda) \lambda^{4} d \lambda}{\int F_{D}(\lambda) d \lambda}
$$

where $F_{D}(\lambda)$ is the fluorescence intensity of the donor in any wavelength $(\lambda)$ from 0 to $\infty$ and $\varepsilon_{A}(\lambda)$ is the extinction coefficient of the acceptor at $\lambda$.

$R_{0}$ was obtained using the following equation [14] (Equation 3):

$$
R_{0}=9.78 \times 10^{3}\left(\mathrm{n}^{-4} Q_{D} \kappa^{2} J(\lambda)\right)^{1 / 6}
$$

where $\mathrm{n}$ is the refrective index, $Q_{D}$ is the quantum yield of the donor, $\kappa^{2}$ is the orientation factor and 2/3 was employed in this study. $J(\lambda)$ must be expressed with $\mathrm{M}^{-1} \mathrm{~cm}^{3}$ to use Equation 3.

\section{Results \& discussion}

In the field of Cell Biology, LSS fluorophores have been used mainly to determine the localization of multiple molecules or to determine multiple biological parameters simultaneously using intramolecular FRET sensors [7-11]. mAmetrine is a bright LSS yellow fluorescence protein, which is efficiently excited by violet light $(\sim 400 \mathrm{~nm})$ and was reported to function well as a FRET donor for tdTomate $[8,12]$. On the other hand, 8-[DY-547]-AET-cAMP is a fluorescent analog of cAMP [15], which has high extinction coefficient (Table 1) with a similar excitation spectrum to that of tdTomate, which was used as the best acceptor of mAmetrine [8]. Therefore, we chose mAmetrine and DY-547 as a new FRET pair to substitute CFP and fluorescein for FRET-based binding assay between the CNBD of human EPAC1 and a fluorescent analog of CAMP [1].

We prepared the CNBD of human

\begin{tabular}{|c|c|c|}
\hline & mAmetrine ${ }^{\dagger} / D Y-547$ [Ref.] & CFP/Fluorescein [Ref.] \\
\hline $\operatorname{Max} e_{D}\left(\mathrm{~cm}^{-1} \mathrm{M}^{-1}\right)$ & 31,000 at $408 \mathrm{~nm}[12]$ & 32,500 at $434 \mathrm{~nm}^{\ddagger}$ \\
\hline$Q_{D}$ & $0.59[12]$ & $0.4[20]^{\S}$ \\
\hline $\operatorname{Max} e_{A}\left(\mathrm{~cm}^{-1} \mathrm{M}^{-1}\right)$ & 150,000 in $\mathrm{EtOH}$ at $559 \mathrm{~nm}^{\pi}$ & 79,000 at $\mathrm{pH} 9$ at $494 \mathrm{~nm}$ \\
\hline$Q_{A}$ & $0.07[17]^{\#}$ & $0.79[21]^{\#}$ \\
\hline$J(\lambda)\left(\mathrm{M}^{-1} \mathrm{~cm}^{3}\right)$ & $6.20 \times 10^{-13+t}$ & $2.56 \times 10^{-13+t}$ \\
\hline$R_{0}(\mathrm{~nm})$ & $6.4^{+\dagger}$ & $5.2^{+\dagger}$ \\
\hline $\mathrm{FI}_{408} / \mathrm{FI}_{\text {max }}(\%)^{\ddagger \ddagger}$ & $0.1^{\dagger \dagger}$ & $2.2^{+\dagger}$ \\
\hline $\mathrm{FI}_{434} / \mathrm{FI}_{494}(\%)$ & - & $6.6^{\dagger \dagger}$ \\
\hline \multicolumn{3}{|c|}{ 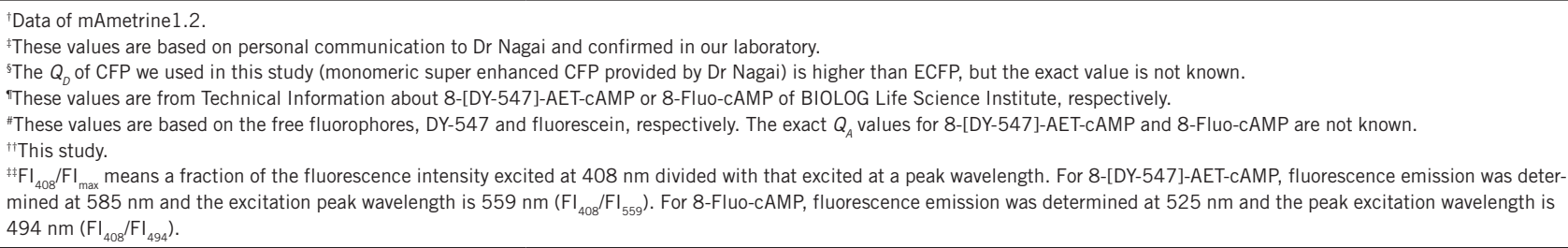 } \\
\hline
\end{tabular}
EPAC1 fused with mAmetrine in the

Table 1. Spectroscopic property of the FRET pairs. 


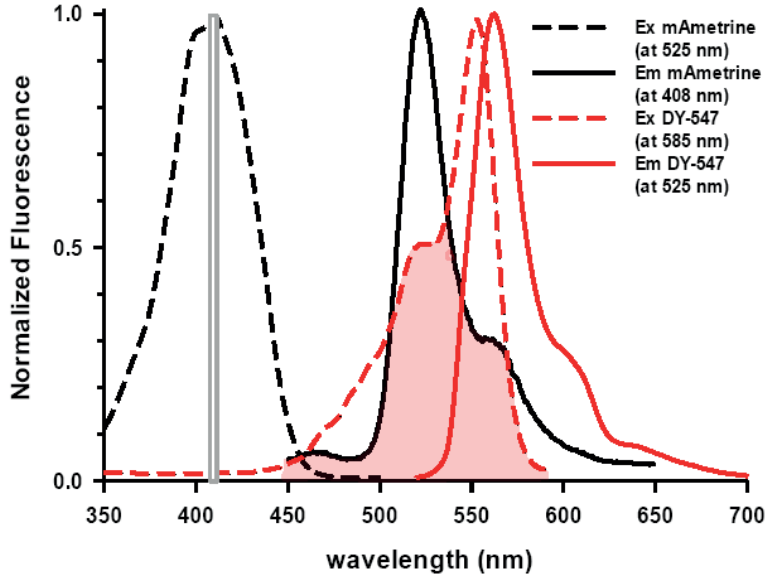

Figure 1. Fluorescence spectra of the new fluorescence resonance energy transfer pair. Normalized fluorescence excitation (dashed line) and emission (solid line) spectra are shown. EPAC1 ${ }_{\text {CBD }}$-mAmetrine (mAmetrine in black) and 8-[DY-547]-CAMP (DY-547 in red). The excitation spectra of mAmetrine and DY-547 were determined using a fixed emission wavelength at $525 \mathrm{~nm}$ and $585 \mathrm{~nm}$, respectively. For the emission spectra, mAmetrine and DY-547 were excited at fixed wavelength, $408 \mathrm{~nm}$ and $525 \mathrm{~nm}$, respectively. In light gray is shown the excitation light (408 nm) used in the subsequent assays. Spectral overlap between mAmetrine excitation and DY-547 emission is indicated by red color.

C terminus (EPAC1 $1_{\mathrm{CNBD}}$-mAmetrine) as described in the Materials \& methods. Figure 1 shows excitation and emission fluorescence spectra of EPAC1 ${ }_{C N B D^{-}}$ mAmetrine and 8-[DY-547]-AET-CAMP. The fusion of the CNBD to mAmetrine did not modify its fluorescence spectra. As shown in the figure, there is an extensive overlap of the emission spectrum of the donor (mAmetrine) and the excitation spectrum of the acceptor (DY-547), an essential requirement of FRET (high value of $J[\lambda]$ in Table 1). On the other hand, most importantly, the shape of the excitation spectrum of 8-[DY-547]-AET-cAMP around the excitation light for the donor (408 nm) is flat (Figure 1 red dashed line and $\mathrm{FI}_{408} /$ $\mathrm{FI}_{\max }$ of $0.1 \%$ in Table 1), which suggests cross-excitation of the acceptor is negligible during the FRET-based binding assay.
As the next step, we performed the FRET-based binding assay using the new FRET pair, EPAC1 $1_{\mathrm{CNBD}}$-mAmetrine and 8-[DY-547]-AET-cAMP (Figure 2). We performed these assays with a fixed donor concentration (50 nM) and three different acceptor concentrations (donor:acceptor ratios of 1:2, 1:5 and 1:10). As expected, the fluorescence intensity of the donor (mAmetrine) was diminished by the addition of the acceptor, 8-[DY-547]-AET-cAMP, and this reduction became more evident with the increasing acceptor concentrations. On the other hand, the increase in fluorescence intensity of the acceptor is small even in high concentrations (1:10 ratio). This is due to the low quantum yield of DY547 [16,17] (Table 1), which is a matter to be improved in the future. In addition, we performed the same experiments in the presence of competitor to prevent FRET and observed that the fluorescence spectra in all conditions were apparently identical to the spectrum of the donor alone. The calculated FRET efficiencies were $0.31 \pm 0.04$, $0.47 \pm 0.06$ and $0.55 \pm 0.04$ (mean \pm S.D.) for donor:acceptor ratios of 1:2, 1:5 and 1:10, respectively. The FRET observed in this experiment is dependent on the specific interaction between the CNBD and CAMP since we did not observe any FRET signal using a mutant of the CNBD with arginine to glutamic acid substitution at the phosphate binding cassette (EPAC1 R279E -mAmetrine) (Figure 3), as previously reported [1].

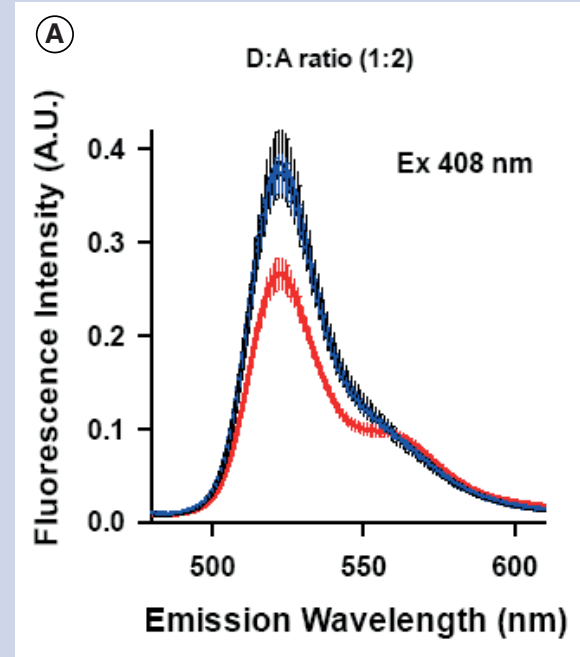

— Donor
(B)

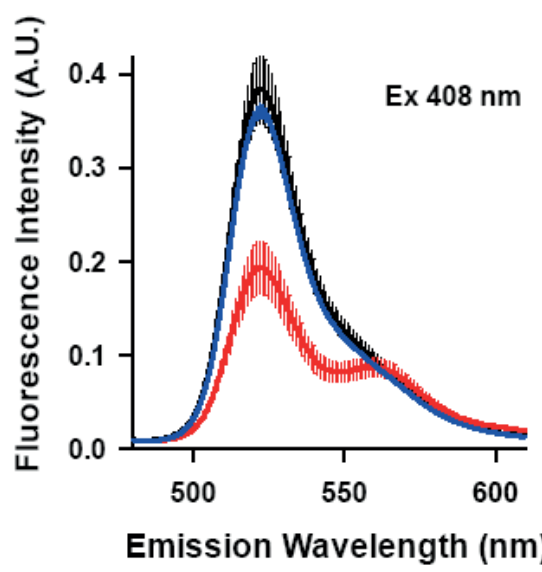

$-\mathrm{D}+$ Acceptor
(C)

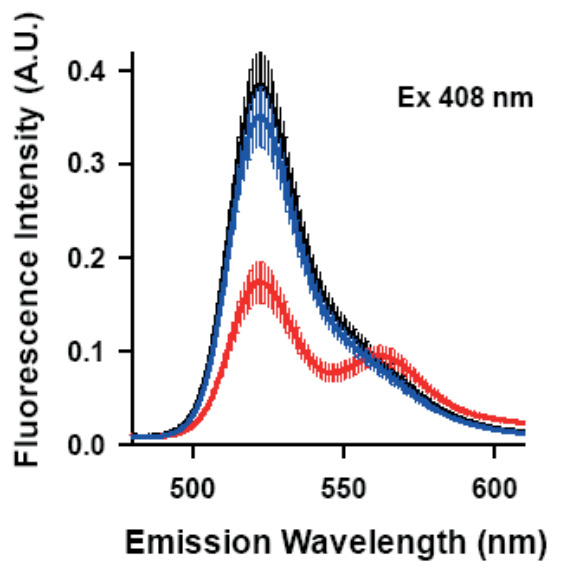

$-D+A+C o m p$

Figure 2. Fluorescence resonance energy transfer (FRET)-based binding assay using the new FRET pair in distinct donor:acceptor ratios. In each panel, black

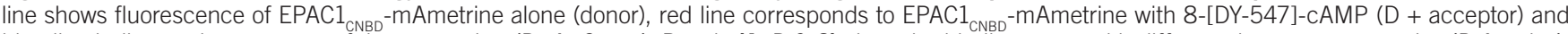
blue line indicates the presence of the competitor $(D+A+C o m p)$. Panels (A, $\mathbf{B} \& \mathbf{C})$ show the binding assay with different donor:acceptor ratios $(D: A$ ratios); $1: 2,1: 5$ and 1:10, respectively. The excitation light used in these assays was $408 \mathrm{~nm}$. Error bars represent the standard deviations. All spectra are the mean of at least three experiments. 


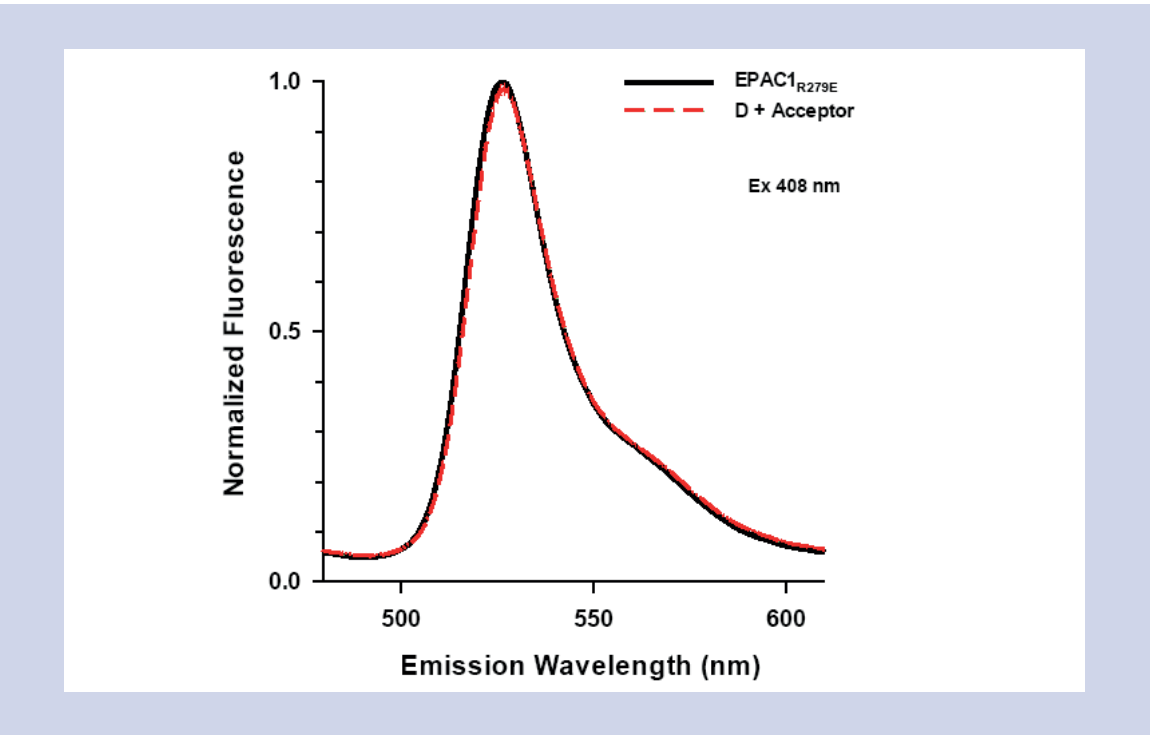

Figure 3. Binding assay with a nonfunctional CNBD. Fluorescence resonance energy transfer-based binding assay was performed with EPAC1 ${ }_{\mathrm{R} 279 \mathrm{E}^{-}}-\mathrm{mAmetrine.} \mathrm{Black}$ line indicates $50 \mathrm{nM}$ of $E P A C 1_{\mathrm{R}^{279 E}}{ }^{-}$

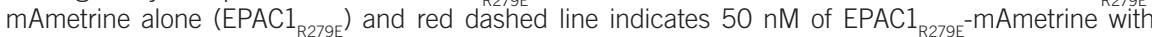
$100 \mathrm{nM}$ of 8-[DY-547]-CAMP (D + Acceptor). No spectral change in EPAC1 ${ }_{\mathrm{R} 279 \mathrm{E}}$-mAmetrine was observed in the absence or the presence of 8-[DY-547]-cAMP. The excitation light used in these assays was $408 \mathrm{~nm}$. All spectra are the mean of at least three experiments.

In order to compare fluorescence spectra between the new and the previous FRET pairs, we performed a similar experiment with EPAC1 ${ }_{\mathrm{CNBD}}$-CFP and 8-FluoCAMP. The fluorescence of the donor (CFP) efficiently decreased by addition of the acceptor (8-Fluo-cAMP) in the absence of the competitor, whereas its fluorescence was almost constant in the presence of the competitor (Figure 4). Conversely, the fluorescence of the acceptor (fluorescein) increased depending on its concentration regardless of the presence or absence of the competitor (Figure 4). Namely, the acceptor was directly excited by the excitation light used for the donor (crossexcitation of the acceptor), not only by FRET as previously shown [1]. Figure 5 shows normalized and superimposed spectra of the two FRET pairs in the presence of the competitor (practically without FRET). As expected, the shape of the fluorescence spectra of the CFP/fluorescein pair varies depending on the donor:acceptor ratio (Figure 5A). By contrast, the shape of the fluorescence spectra of mAmetrine/ DY-547 pair is constant independently of donor:acceptor ratio (Figure 5B). Therefore, we can rapidly and easily estimate the occurrence of FRET (molecular interaction) between the EPAC1 ${ }_{\mathrm{CNBD}}$ and CAMP analog using the new FRET pair (mAmetrine/ DY-547) compared with previous FRET pair (CFP/fluorescein) [1].

In our previous report, we observed that 8-Fluo-cAMP has much higher affinity $(6 \mathrm{nM})$ to the isolated CNBD of EPAC1 [1] than intact cAMP $(4 \mu \mathrm{M})$ [18]. To determine the affinity of 8-[DY-547]-AET-CAMP to the CNBD, we performed the binding assays using a fixed ratio of EPAC ${ }_{C N B D}-m A m e t r i n e$ and 8-[DY-547]-AET-CAMP (1:2) but with varied concentrations by serial dilution as previously established [1]. As shown in Figure $6 \mathrm{~A}$, the shapes of fluorescence emission spectra as well as absolute fluorescence intensities vary depending on the absolute concentration of $\mathrm{EPAC}_{\mathrm{CNBD}}-\mathrm{mAmetrine}$. The normalized fluorescence spectra at the isoemissive point ( $563 \mathrm{~nm}$ ) clearly show the changes of spectral shapes (Figure 6B), which directly indicate the changes of the FRET degrees and namely the changes of the binding status (Figure 6C). The
(A)

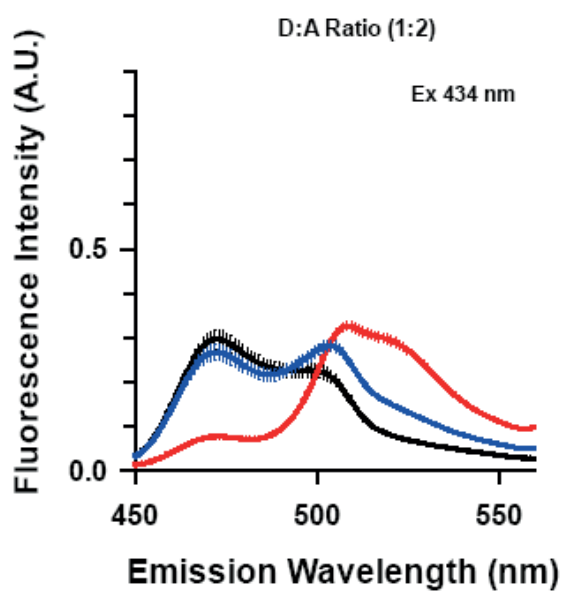

(B)

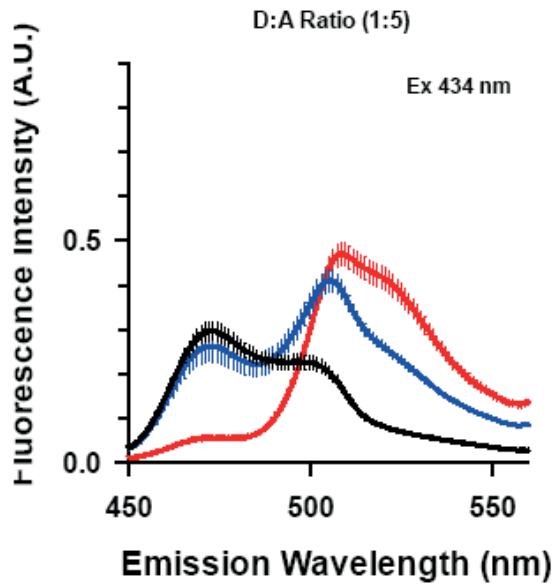

(C)

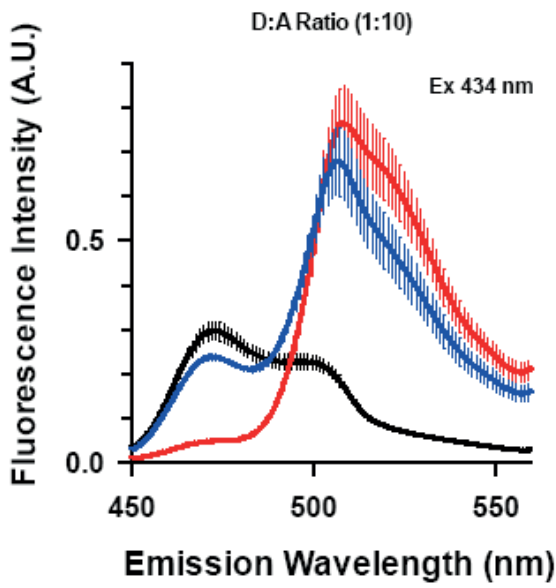

Figure 4. Fluorescence resonance energy transfer-based binding assay using CFP/Fluorescein pair in distinct donor:acceptor ratios. In each panel, black line shows the fluorescence of EPAC1 ${ }_{C N B D}-C F P$ alone, red line corresponds to EPAC1 ${ }_{C N B D}-C F P$ plus 8-Fluo-CAMP (D+8-Fluo-CAMP) and blue line indicates the presence the competitor ( $\mathrm{D}+\mathrm{A}+\mathrm{Comp})$. Panels (A, B \& $\mathbf{C}$ ) show the binding assay with different donor:acceptor ratios: 1:2, 1:5 and 1:10, respectively. In each condition, $100 \mathrm{nM}$ of the donor was used and the concentration of the competitor (8-CPT-cAMP) was 100 times-higher than that of acceptor (not enough to compete 100\%). The excitation light used in these assays was $434 \mathrm{~nm}$. Error bars represent the standard deviations. All spectra are the mean of at least three experiments. 


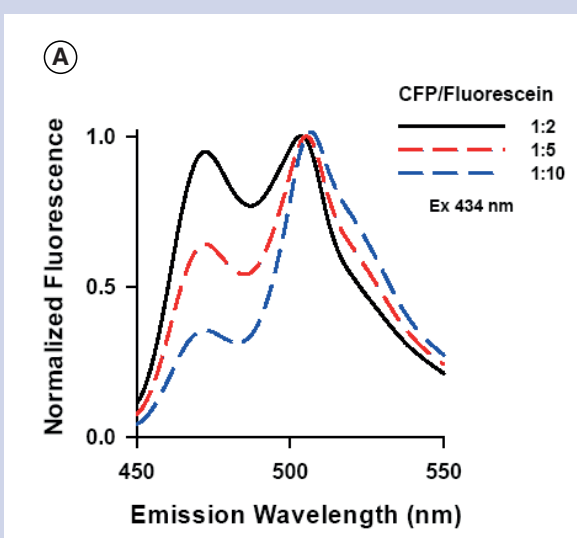

(B)

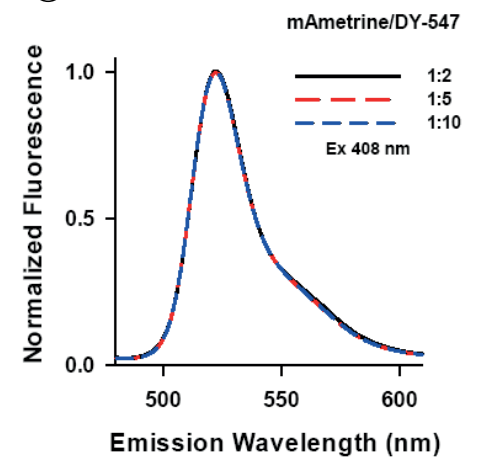

Figure 5. Normalized spectra of two fluorescence resonance energy transfer pairs with different donor:acceptor ratios. Panels (A \& B) show normalized spectra in the presence of the competitor (without fluorescence resonance energy transfer) of Figure 4 (CFP/fluorescein) excited at $434 \mathrm{~nm}$ and Figure 2 (mAmetrine/DY-547) excited at $408 \mathrm{~nm}$, respectively. Donor:Acceptor ratio, 1:2 (black line), 1:5 (Red dashed line) and 1:10 (blue dashed line).

calculated $K_{d}$ value between EPAC $_{\mathrm{CNBD}^{-}}$ mAmetrine and 8-[DY-547]-AET-CAMP is $713 \pm 146 \mathrm{nM}$. This value is much larger than that obtained for 8-Fluo-cAMP (6 nM) [1], but still lower than intact CAMP and similar to those of other cAMP with some modification at the position 8 such as 8-CPTcAMP (250 nM) [1]. The maximum FRET efficiency between EPAC $_{\mathrm{CNBD}}$-mAmetrine and 8-[DY-547]-AET-CAMP is approximately $75 \%$. This value is smaller than the previous FRET pair CFP/Fluorescein (>80 \%) although the calculated Förster distance of mAmetrine/DY-547 pair is larger than that of CFP/fluorescein (Table 1). This discrepancy could be partially explained by the difference of donor quantum yield $\left(Q_{D}\right)$ between the
ECFP (0.37) and the CFP we used (Table 1). Alternative reasons such as an orientation factor (it may be $\kappa^{2} \neq 2 / 3$ ) in each condition may also account for the difference besides the difference of $Q_{D}$.

We tried to determine lifetime of the donor fluorescence to corroborate the FRET efficiency of mAmetrine/DY-547 pair. However, we obtained longer lifetimes than those expected since the band-pass filters we used in our set-up were not appropriate (data not shown). In other words, we failed to determine the exact lifetime of the donor fluorescence owing to the bleed-through emission of the acceptor. In this sense, our new FRET pair is good at avoiding crossexcitation of the acceptor, but it does not prevent the cross-talk of the fluorescence emission between the donor and the acceptor. Taking this limitation into account, FRET using two LSS fluorophores for both donor and acceptor [19] would be an ideal combination with more robust determination of FRET efficiency particularly when we use a band-pass filter to detect the donor fluorescence intensity instead of a monochromator.

In conclusion, mAmetrine and DY-547 work well to perform an intermolecular FRET assay because the excitation light used for mAmetrine (approximately $400 \mathrm{~nm}$ ) does not directly excite DY-547; namely, there is practically no cross-excitation of the acceptor (Table 1). Considering this experimental advantage, the new FRET pair, mAmetrine/DY-547, might be useful to study other types of molecular interactions, such as receptor-ligand and antibodyantigen interactions. In principle, use of any LSS-FPs as FRET donors should reduce the cross-excitation of the acceptor in most of the FRET pairs currently used. Therefore, we should consider substituting a normal FP by a LSS-FP as a FRET donor to perform any intermolecular FRET experiments.

\section{Author contributions}

TN conceived the project. CSC performed most of the experiments. FR and ILG determined $\mathrm{Kd}$ value between $\mathrm{EPAC1}$ CNBD $^{-}$ mAmetrine and 8-[DY-547]-AET-CAMP. CSC and TN wrote the manuscript. CSC
(A)

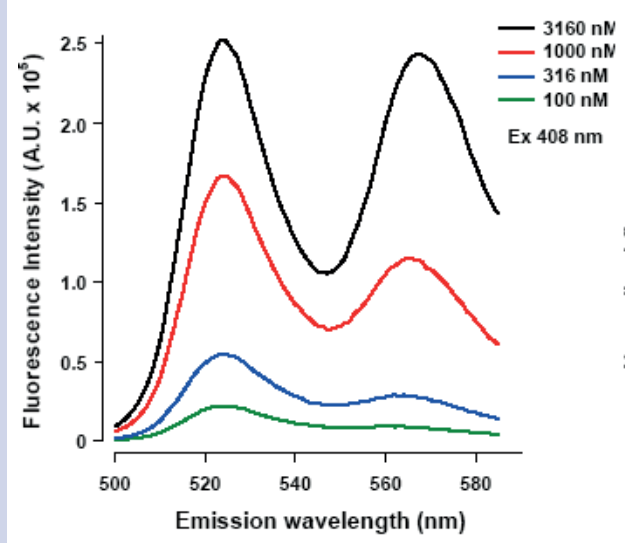

(B)

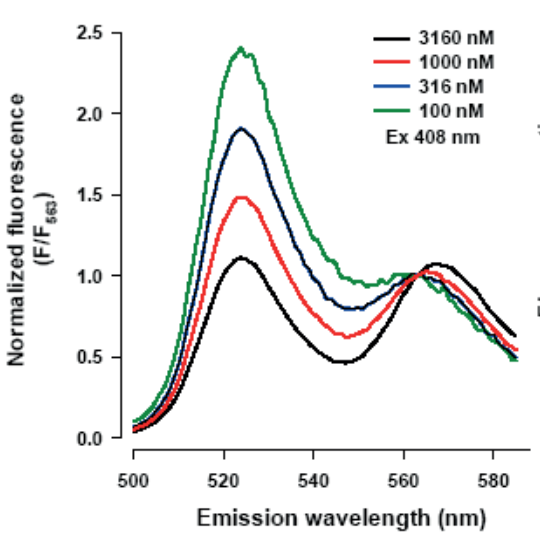

(c)

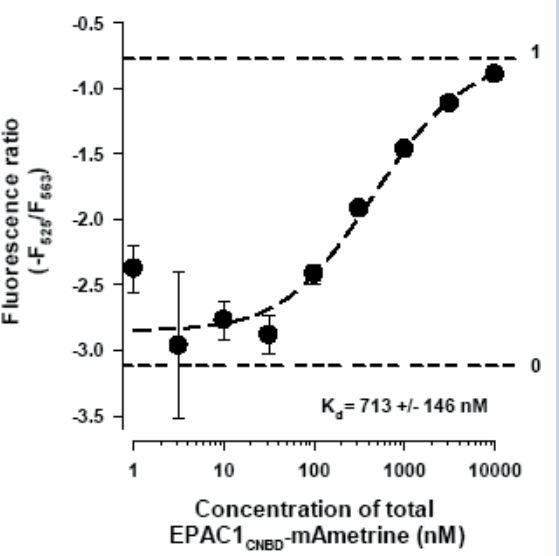

Figure 6. Determination of $\mathrm{K}_{\mathrm{d}}$ between EPAC1 $1_{\text {CNBD }}$-mAmetrine and 8-[DY-547]-CAMP in equilibrium condition. Panel (A) shows representative fluorescence

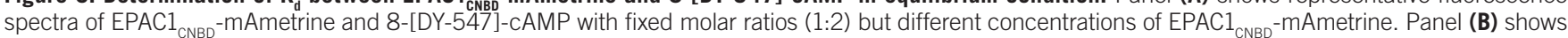
normalized fluorescence spectra of the panel $(\mathbf{A})$ using fluorescence intensities of the isoemissive point (563 nm). Panel (C) represents the relative fluorescence resonance energy transfer efficiencies $\left(\mathrm{F}_{525} / \mathrm{F}_{563}\right)$ in the function of total concentration of EPAC1 $1_{C N B D}-\mathrm{mAmetrine}$. The $K_{d}$ value between EPAC1 ${ }_{\mathrm{CNBD}}{ }^{-}$ mAmetrine and 8-[DY-547]-cAMP was calculated by three independent experiments. The fraction of the bound CNBD is indicated with dashed lines from 0 to 1 . The excitation wavelength used in this experiment was $408 \mathrm{~nm}$. 
and ILG prepared the figures. All authors revised and approved the manuscript.

\section{Acknowledgments}

We thank Dr Robert Campbell for providing pBAD-mAmetrine1.2. We appreciate the National Institute of Genetics for providing two E. coli strains, JM109 (DE3) and BL21 (DE3). We thank Eugenio López, Jorge Yáñez and Paul Gaytán for oligonucleotide synthesis and plasmid sequencing. We also thank Dr. Alejandra Covarrubias for sharing the FS5 Spectrofluorometer with SC-40.

\section{Financial \& competing interests disclosure}

This work was supported by the following grants: CONACYT-Ciencia Básica 177138 (TN), 128566 (Consorcio de fisiología del espermatozoide), CONACYT-Fronteras de la ciencia 71, UNAM/DGAPA-PAPIIT IN203513 (TN), IN206116 (TN), IN205518 (ILG). The authors have no other relevant affiliations or financial involvement with any organization or entity with a financial interest in or financial conflict with the subject matter or materials discussed in the manuscript apart from those disclosed.

No writing assistance was utilized in the production of this manuscript.

\section{Open access}

This work is licensed under the Attribution-NonCommercial-NoDerivatives 4.0 Unported License. To view a copy of this license, visit http://creativecommons.org/ licenses/by-nc-nd/4.0/

\section{Supplementary data}

To view the supplementary data that accompany this paper please visit the journal website at: www.future-science. com/doi/suppl/10.2144/btn-2018-0041

\section{References}

1. Romero F, Santana-Calvo C, Sánchez-Guevara $Y$, Nishigaki T. FRET-based binding assay between a fluorescent CAMP analogue and a cyclic nucleotide-binding domain tagged with a CFP. FEBS Letters 591, 2869-2878 (2017).

2. Scott SP, Shea PW, Dryer SE. Mapping ligand interactions with the hyperpolarization activated cyclic nucleotide modulated ( $\mathrm{HCN}$ ) ion channel binding domain using a soluble construct. Biochemistry 46(33), 9417-9431 (2007).

3. Tsalkova T, Mei FC, Cheng X. A fluorescencebased high-throughput assay for the discovery of exchange protein directly activated by cyclic AMP (EPAC) antagonists. PloS One 7(1), e30441 (2012).

4. Piston DW, Kremers G-J. Fluorescent protein FRET: the good, the bad and the ugly. Trends Biochem. Sci. 32(9), 407-414 (2007).

5. Zal T, Gascoigne NRJ. Photobleachingcorrected FRET efficiency imaging of live cells. Biophysical. J. 86(6), 3923-3939 (2004).

6. Erickson MG, Alseikhan BA, Peterson BZ, Yue DT. Preassociation of calmodulin with voltagegated $\mathrm{Ca}^{2+}$ channels revealed by FRET in single living cells. Neuron 31, 973-985 (2001).

7. Zapata-Hommer O, Griesbeck O. Efficiently folding and circularly permuted variants of the Sapphire mutant of GFP. BMC Biotechnol. 3(1), 5 (2003).

8. Ai H-W, Hazelwood KL, Davidson MW, Campbell RE. Fluorescent protein FRET pairs for ratiometric imaging of dual biosensors. Nat. Methods 5(5), 401-403 (2008).

9. Laviv T, Kim BB, Chu J, Lam AJ, Lin MZ, Yasuda R. Simultaneous dual-color fluorescence lifetime imaging with novel red-shifted fluorescent proteins. Nat. Methods 13(12), 989-992 (2016).

10. Shcherbakova DM, Hink MA, Joosen L, Gadella TWJ, Verkhusha VV. An orange fluorescent protein with a large Stokes shift for singleexcitation multicolor FCCS and FRET imaging. J. Am. Chem. Soc. 134(18), 7913-7923 (2012).

11. Niino Y, Hotta K, Oka K. Simultaneous live cell imaging using dual FRET sensors with a single excitation light. PloS One 4(6), e6036 (2009).

12. Ding $\mathrm{Y}, \mathrm{Ai} \mathrm{H}$, Hoi H, Campbell RE. Förster resonance energy transfer-based biosensors for multiparameter ratiometric imaging of $\mathrm{Ca}^{2+}$ dynamics and caspase- 3 activity in single cells. Anal. Chem. 83(24), 9687-9693 (2011).
13. Heim R, Prasher DC, Tsien RY. Wavelength mutations and posttranslational autoxidation of green fluorescent protein. Proc. Natl Acad. Sci. USA 91(26), 12501-12504 (1994).

14. Lakowicz JR. Energy transfer. In: Principles of Fluorescence Spectroscopy. Springer, 443-475 (2006).

15. Kusch J, Biskup C, Thon S et al. Interdependence of receptor activation and ligand binding in HCN2 pacemaker channels. Neuron 67(1), 75-85 (2010)

16. Cooper M, Ebner A, Briggs $\mathrm{M}$ et al. CyзB (TM): Improving the performance of cyanine dyes. $\mathrm{J}$. Fluorescence 14(2), 145-150 (2004).

17. Linck L, Kapusta P, Resch-Genger U. Spectroscopic and photophysical properties of dUTP and internally DNA bound fluorophores for optimized signal detection in biological formats. Photochem. Photobiol. 88(4), 867-875 (2012).

18. De Rooij J, Rehmann H, Van Triest M, Cool $\mathrm{RH}$, Wittinghofer A, Bos JL. Mechanism of regulation of the Epac family of CAMP-dependent RapGEFs. J. Biol. Chem. 275(27), 20829-20836 (2000).

19. Liu Z, Song F, Song B et al. A FRET chemosensor for hypochlorite with large Stokes shifts and long-lifetime emissions. Sens. Actuators B. Chem. 262, 958-965 (2018).

20. Cubitt AB, Woollenweber LA, Heim R. Chapter 2: Understanding structure-function relationships in the aequorea victoria green fluorescent protein. In: Green Fluorescent Proteins. Sullivan KF, Kay SA (Eds). Elsevier, NY, USA, 19-30 (1998).

21. Kellogg RE, Bennett RG. Radiationless intermolecular energy transfer. III. Determination of phosphorescence efficiencies. J. Chem. Phys. 41(10), 3042-3045 (1964).

First draft submitted: 9 April 2018; Accepted for publication: 13 July 2018

Address correspondence to: Takuya Nishigaki, Departamento de Genética del Desarrollo y Fisiología Molecular, Instituto de Biotecnología, Universidad Nacional Autónoma de México (IBT, UNAM), Avenida Universidad 2001, Col. Chamilpa, C.P. 62210,

Cuernavaca, Morelos, México; takuya@ibt.unam.mx

To purchase reprints of this article contact: s.cavana@future-science.com 